\title{
Perceptual and Social Challenges in Body Proximate Display Ecosystems
}

$\begin{array}{ll}\text { Aaron Quigley } & \text { Jens Grubert } \\ \text { SACHI } & \text { University of Passau } \\ \text { School of Computer Science } & \text { Passau, Germany } \\ \text { University of St Andrews, UK } & \text { jg@jensgrubert.de } \\ \text { aquigley@acm.org } & \end{array}$

Permission to make digital or hard copies of part or all of this work for personal or classroom use is granted without fee provided that copies are not made or distributed for profit or commercial advantage and hat coples bear this notice and the full citation For all Mor all

http://dx.doi.org/10.1145/2786567.2794349

\begin{abstract}
Coordinated multi-display environments from the desktop, second-screen to gigapixel display walls are increasingly common. Personal and intimate display devices such as head-mounted displays, smartwatches, smartphones and tablets are rarely part of such a multi-display ecosystem. This presents an opportunity to realise "body proximate" display environments, employing on and around the body displays. These can be formed by combining multiple handheld, head-mounted, wrist-worn or other personal or appropriated displays. However, such an ecosystem encapsulating evermore interaction points, is not yet well understood. For example, does this trap the user in an "interaction bubble" even more than interaction with individual displays such as smartphones? Within this paper, we investigate the perceptual and social challenges that could inhibit the adoption and acceptance of interactive proximate display ecosystems. We conclude with a series of research questions raised in the consideration of such environments.
\end{abstract}

\section{ACM Classification Keywords}

H.5.m [Information interfaces and presentation]: Graphical user interfaces

\section{Introduction}

Multi-display systems have emerged as a mainstay for knowledge work (eg. programming or financial trading) and 


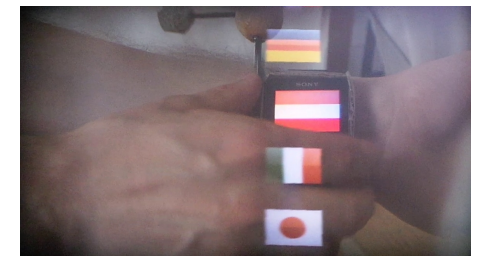

Figure 1: Multifi [2], head-mounted and smartwatch coupled display extending the screen space of the smartwatch

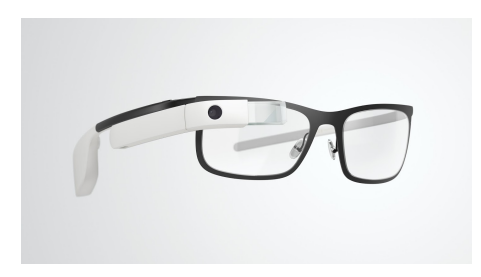

Figure 2: Google Glass

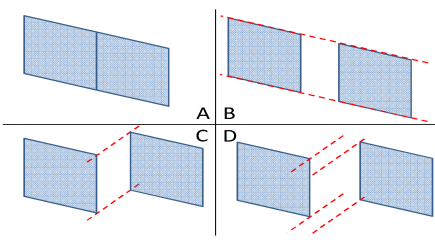

Figure 3: Display contiguity factor [8]. A: visual-field (VF) and Depth (DE) contiguous. B: VF discontiguous and DE contiguous. C: VF contiguous and DE discontiguous. D: both discontiguous. complex tasks (eg. city or factory management) [12]. Socia applications such as gaming and second screen experiences are further extending the proliferation of increasingly complex display ecosystems. Today, we see the emergence of another range of more mobile and intimate display technology, from wrist-worn smartwatches and health devices (eg. fitbit or Microsoft Band as shown in Figure 4) to headmounted displays [2] (see Figures 2 and 5) or personal devices such as smartphones and tablets. A motivating question for us here is, can these disparate forms of displays be coupled and coordinated to realise "proximate display environments", with on and around the body interaction as shown in Figure 1 from [2]?

Of course, once we start to use an ecosystem of connected and coupled personal or appropriated displays (as shown in Figure 4), a number of challenges arise. How will we manage our attention, visual focus or awareness as our interaction is being spread across an ever wider display ecosystem? Which of our elements of human perception will this challenge? And will this form of interaction raise serious social challenges for us to face? These are the issues we seek to begin to address in this paper as distinct from the design and technology issues [7] introduced elsewhere.

\section{Perceptual Challenges}

There are numerous challenges with human perception in body proximate display ecosystems from physiological to cognitive levels. Such issues stem from varying display resolutions, luminance, effective visual fidelities, visual interference, color or contrast in display overlap which can be experienced with body proximate ecosystems. Here we focus on the issues of display switching, focus (or accomodation) and field of view.
Display Switching Existing research has identified the cost of display switching [9] and the factors which influence visual attention in multi-display user interfaces [8]. These factors include display contiguity (as shown in Figure 3), angular coverage, content coordination, input directness and input-display correspondence. Further physiological issues arise with depth discontiguous displays, field of view, visual acuity or indeed blind spots with monocular displays employed in multi-display interaction.

Focus in Human Vision The shape of our lens and iris alters how much light enters and how our eyes focus. However, our eyes cannot focus sharply on two displays which are near and far simultaneously. If the virtual display plane of an optical see-through head-mounted device is in sharp focus, then effectively closer or distant displays won't be. This can be easily seen with a smartwatch which is in focus but is then surrounded by unfocused input from displays effectively further from the eye. The effective distance, not actual distance, needs to be considered as devices, such as optical see-through displays (eg. Google Glass) often employ optical techniques to generate images at distances which are easier for the eye to accomodate. A further issue to consider is that as the ciliary muscles in our eyes age, our range of accommodation declines. As a result, with body proximate ecosystems we can experience a mixed focus interface with some elements in sharp focus while others are defocused. This is quite unlike typical display environments, where each element of the display is typically in focus.

Another byproduct of our eyes inability to focus sharply on two distances, is that it then takes time for the eye to refocus on objects at different distances. In addition, the speed of this process also declines as the muscles age. However, with body proximate ecosystems the eye will need notice- 


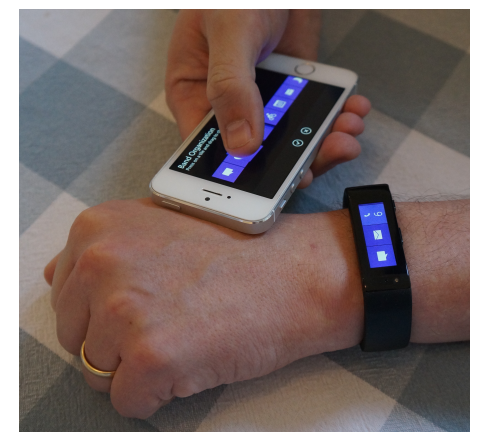

Figure 4: Microsoft Band and Health Application on iPhone

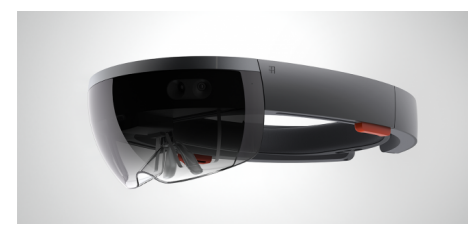

Figure 5: Microsoft HoloLens able amounts of time (eg. $300 \mathrm{msec}$ latency and $1000 \mathrm{msec}$ stabilisation period [1]) for the focal power of the eye to adapt in markedly discontiguous display spaces. Further, these accomodation times don't include movements if the displays are "visually field discontiguous" [9].

Field of View Humans have a limited field of view and an age diminished "useful field of view" (UFOV) [11], which needs to be considered. Excluding head rotation, the typical field of view for a human has a difference between the horizontal and vertical field of view, an area of binocular overlap and areas of monocular far peripheral vision. "For many of our interaction tasks the UFOV varies between younger and older people. A 36 degree field of view will be practical in many situations [11]. Within each person's field of view we can also distinguish regions of central (ie. foveal, central, paracentral and macular) and peripheral (near, mid and far) vision. The useful field of view above, typically includes both central and largely near peripheral parts of vision.

\section{Social Challenges}

Beyond our personal perception of the world, there are a number of social challenges that body proximate ecosystems systems present, including privacy, social acceptability, social participation, social exclusion and social engagement.

Privacy presents a major challenge in the use of public or semi-public displays as part of a wearable multi-display ecosystem. We can consider such forms of social interaction with technology at different scales from inch to chain and beyond [12] as shown in Figure 6. Personal devices overcome the privacy challenge by use of private environments, use at an intimate distance, privacy screens or non-visual modalities. Questions arise when we consider

\begin{tabular}{|c|c|c|c|c|c|}
\hline $\begin{array}{l}\text { Social Scale } \\
\text { Interaction }\end{array}$ & Inch & Foot & Yard & Perch & Chain \\
\hline One-One & Shake & \begin{tabular}{|c|c|c|} 
Connectablele \\
Ubitable
\end{tabular} & Touch/Push & Proxnet & Pong \\
\hline One-Few & & Bump & $\begin{array}{l}\text { Point\& } \\
\text { Shoot }\end{array}$ & Map & \\
\hline Few-Few & & Stitch & \begin{tabular}{|l|} 
Point-Tap \\
BlueTable \\
\end{tabular} & Proxnet & \\
\hline One-Many & & & & & Pong \\
\hline Many-Many & & & & \\
\hline
\end{tabular}

Figure 6: Multi-person-display ecosystem taxonomy [12], social interaction support and ecosystem scale

how we might share content on intimate displays, at varying scales, different social interaction types or even share content spanning multiple private displays.

Social acceptability The use of wearable on body displays presents a range of social acceptability issues. Some of the inherent form factors (eg. Microsoft Hololens in Figure 5) can present acceptability challenges. In addition, existing research has explored the suitability of different parts of the body for gestural inputs, along with issues of social norms and behaviour [6]. Here, body proximate displays introduce new challenges as the coordination and movement of multiple displays can require unusual inter-display coordination and body orientation. Also, in contrast to touch-only operated displays such as smartphones, the manipulations of multiple body proximate displays through spatial gestures are more visible whereas the effects of those actions remain hidden to bystanders [10]. Depending on the social situation this could lead to inhibited or non-use of an interactive system, similar to observations made for handheld Augmented Reality systems [3, 4]. Further issues arise 
from the use of shared or public display elements within an ecosystem. All of these issues are modulated by difference cultures, work practices, age, familiarity with technology an evolving social norms for new technology behaviours.

Social participation Today, civic discourse is impacted by the isolation that technologies provide people. For example, the "filter bubble [5]" stems from the personalisation in search results presented to individual people. Such bubbles can socially isolate people from one another, into their own economic, political, cultural and hence ideological groups. With body proximate ecosystems, we might further encourage people into "interaction bubbles" which isolates them further from others and discourages interpersonal interaction. The "in-your-face nature" of what is proposed, is unlike other forms of technology.

Social exclusion Mirroring the problems in social participation are the further challenges of social exclusion. By augmenting our interactions with body proximate ecosystems we are changing the nature of our interaction with the world. Many personal technologies reside out of sight, whereas wearable and on body displays present a visible digital alienation to those without access to such technology. By allowing some to see and experience more than others can see are we further disenfranchising people? Do these technologies further reinforce a digital social stratification we are already witnessing?

Social engagement In using semipublic or public displays as part of an egocentric body proximate ecosystem issues of performance and social engagement present themselves. These challenges are also opportunities for improved social engagement between people but also draw into question the appropriateness of any device appropriation. Fair use, sharing space or time, along with the use of non-visual modalities present challenges for the design and deployment of such systems.

\section{Research Questions}

There are a number of sociotechnical research questions which arise in the consideration of body proximate display ecosystems. Some of the provocative and prosaic ques-

tions we propose for discussion in this workshop and for the future include:

- Many people clearly crave the ability to isolate themselves from one another in "interaction bubbles". Are body proximate display ecosystems the best way to help them do this?

- With the close distances inherent in such displays, are we straining our perceptual capabilities (field of view, vision, switching) beyond what is comfortable and usable?

- Do the interaction bubbles we create with these ecosystems magnify issues of privacy, social acceptability, participation and exclusion? In effect, are we designing inherently rude technologies?

- Can we become fluent with such forms of interaction so that our social situations are enhanced, instead of negatively impacted?

- Are body proximate display devices destined to be data silos? Will each be a "island of interaction" or is a fragmented "archipelago of interaction" the best we might hope for? Or can a true coordinated device ecosystem be realised?

- Do the different characteristics, functions, features and modalities employed with body proximate devices 
imply an explosion of interaction pathways which cannot be accommodated in general but rather only in specialised cases?

- How do the social and perceptual factors of interaction impact on the design factors or interface widgets [7]?

- Do the sensing and cross-device development issues outweigh the social and perceptual challenges [7]? If a device ecosystem could be easily sensed and aligned, and if operating systems or middle-ware supported it, would this solve the major challenges in this area?

These questions cut across challenges introduced in this paper and other research [7].

\section{Conclusion}

Within this paper we have considered some of the social and perceptual challenges and questions raised in interaction with multiple displays on and around the body, including smartwatches, smartglasses and smartphones. Body proximate display applications give rise to a range of other issues which challenge the limits of the human being and our social sphere at varying scales (see Figure 6). The social challenges may simply be opportunities while adapting our display environments to accommodate for our field of view, current focus and the cost of display switching are just some of the perceptual challenges we face. Through discussing these challenges and questions we hope to contribute to shaping the research agenda of interaction with multi-display environments on and around the body ie. body proximate display ecosystems. 


\section{REFERENCES}

1. W Neil Charman. 2008. The eye in focus: accommodation and presbyopia. Clinical and Experimental Optometry 91, 3 (2008), 207-225. DOI : http://dx.doi.org/10.1111/j.1444-0938.2008.00256.x

2. Jens Grubert, Matthias Heinisch, Aaron Quigley, and Dieter Schmalstieg. 2015. MultiFi: Multi Fidelity Interaction with Displays On and Around the Body. In Proceedings of the 33rd Annual ACM Conference on Human Factors in Computing Systems (CHI '15). ACM, New York, NY, USA, 3933-3942. DOI :

http://dx.doi.org/10.1145/2702123.2702331

3. Jens Grubert, Ann Morrison, Helmut Munz, and Gerhard Reitmayr. 2012. Playing it real: magic lens and static peephole interfaces for games in a public space. In Proceedings of the 14th international conference on Human-computer interaction with mobile devices and services. ACM, 231-240. DOI :

http://dx.doi.org/10.1145/2371574.2371609

4. Jens Grubert and Dieter Schmalstieg. 2013. Playing it real again: a repeated evaluation of magic lens and static peephole interfaces in public space. In Proceedings of the 15th international conference on Human-computer interaction with mobile devices and services. ACM, 99-102. DOI:

http://dx.doi.org/10.1145/2493190.2493234

5. Eli Pariser. 2011. The Filter Bubble: What the Internet Is Hiding from You. Penguin Group.

6. Halley P. Profita, James Clawson, Scott Gilliland, Clint Zeagler, Thad Starner, Jim Budd, and Ellen Yi-Luen Do. 2013. Don'T Mind Me Touching My Wrist: A Case Study of Interacting with On-body Technology in Public. In Proceedings of the 2013 International Symposium on Wearable Computers (ISWC '13). ACM, New York, NY, USA, 89-96. DOI :

http://dx.doi.org/10.1145/2493988.2494331

7. Aaron Quigley, Matthias Kranz, and Jens Grubert. 2015. Design and Technology Challenges for Body Proximate Display Ecosystems. In MobileHCl'15 Adjunct, ACM (Ed.). DOI :

http://dx.doi.org/10.1145/2786567.2794310

8. Umar Rashid, Miguel A. Nacenta, and Aaron Quigley. 2012a. The Cost of Display Switching: A Comparison of Mobile, Large Display and Hybrid UI Configurations. In Proceedings of the International Working Conference on Advanced Visual Interfaces (AVI '12). ACM, New York, NY, USA, 99-106. DOI : http://dx.doi.org/10.1145/2254556.2254577

9. Umar Rashid, Miguel A. Nacenta, and Aaron Quigley. 2012b. Factors Influencing Visual Attention Switch in Multi-display User Interfaces: A Survey. In Proceedings of the 2012 International Symposium on Pervasive Displays (PerDis '12). ACM, New York, NY, USA, Article 1, 6 pages. DOI :

http://dx.doi.org/10.1145/2307798.2307799

10. Stuart Reeves, Steve Benford, Claire O'Malley, and Mike Fraser. 2005. Designing the spectator experience. In Proceedings of the ACM Conference on Human Factors in Computing Systems. ACM, 741-750. DOI : http://dx.doi.org/10.1145/1054972.1055074

11. Eric Richards, Patrick J. Bennett, and Allison B. Sekuler. 2006. Age related differences in learning with the useful field of view. Vision Research 46, 25 (2006), 4217 - 4231. DOI :

http://dx.doi.org/doi:10.1016/j.visres.2006.08.011 
12. Lucia Terrenghi, Aaron Quigley, and Alan Dix. 2009. A Taxonomy for and Analysis of Multi-person-display

Ecosystems. Personal Ubiquitous Comput. 13, 8 (Nov.

2009), 583-598. DOI :

http://dx.doi.org/10.1007/s00779-009-0244-5 